\title{
Avaliação da satisfação de usuários a partir da Carta SUS, no Rio Grande do Norte, Brasil
}

\author{
Evaluation of user satisfaction from the Carta SUS, in Rio Grande do \\ Norte, Brazil
}

Danyllo do Nascimento Silva Junior', Bárbara Suellen Fonseca Braga², Camila Dayze Pereira Santos ${ }^{3}$, Raul Elton Araújo Borges ${ }^{4}$, Luiz Roberto Augusto Noro ${ }^{5}$

1 Universidade Federal do Rio Grande do Norte (UFRN), Programa de Pós-Graduação em Saúde Coletiva (PPGSCol) - Natal (RN), Brasil.

Orcid: https://orcid org/0000-0002-90356216

danyllojunior@hotmail.com

2 Universidade Federal do Rio Grande do Norte (UFRN), Programa de Pós-Graduação em Saúde Coletiva (PPGSCol) - Natal (RN), Brasil. Orcid: https://orcid. org/0000-0002-66589976

babis.f.b@gmail.com

3 Universidade Federal do Rio Grande do Norte (UFRN), Programa de Pós-Graduação em Saúde Coletiva (PPGSCol) - Natal (RN), Brasil.

Orcid: https://orcid. org/0000-0001-80922846

camila_nutufrn@hotmail. com

4 Universidade Federal do Rio Grande do Norte (UFRN), Faculdade de Ciências da Saúde do Trairi (Facisa) - Natal (RN),

Brasil.

Orcid: https://orcid. org/0000-0003-38616890

raullelton@hotmail.com

5 Universidade Federal do Rio Grande do Norte (UFRN), Programa de Pós-Graduação em Saúde Coletiva (PPGSCol) - Natal (RN), Brasil.

Orcid: https://orcid. org/0000-0001-82440154

luiz_noro@hotmail.com
DOI: 10.1590/0103-1104201811909

RESUMO Objetivou-se analisar a satisfação dos usuários e a caracterização dos serviços de atendimento hospitalar e ambulatorial do Sistema Único de Saúde (SUS). Realizou-se um estudo transversal analítico por meio de dados de usuários que responderam ao instrumento Carta SUS entre 2012 e 2013, no estado do Rio Grande do Norte, no qual foi avaliada a qualidade da estrutura física, da equipe de saúde e do tratamento ofertado. Dos 17.333 usuários que responderam à Carta SUS no período estudado, 62,3\% eram mulheres e 62,4\% tinham acima de 40 anos. A maior parte dos atendimentos $(56,8 \%)$ foi realizada na capital e em serviços públicos (70,3\%). Os usuários apresentaram-se satisfeitos com os serviços, com um percentual que variou de 79 a $90 \%$, de acordo com as regiões de saúde. Os homens e as pessoas com mais de 60 anos avaliaram mais positivamente os serviços; a alta complexidade e os serviços da capital tiveram melhores resultados $(\mathrm{p} \leq 0,001)$, assim como as unidades privadas. Ressaltam-se a importância da avaliação em saúde e a relevância de se analisar, divulgar e dar continuidade ao instrumento Carta SUS, com vistas a subsidiar mais estudos e reflexões sobre a qualidade dos serviços.

PALAVRAS-CHAVE Avaliação em saúde. Assistência hospitalar. Assistência ambulatorial. Satisfação dos usuários.

ABSTRACT The purpose of this study was to analyze user satisfaction and the characterization of the hospital and outpatient services of the Unified Health System (SUS). A cross-sectional analytical study was performed using user data that responded to the Carta SUS instrument between 2012 and 2013, in the state of Rio Grande do Norte, in which the quality of the physical structure, the health team and the treatment offered. Of the 17.333 users who answered the SUS Charter in the period studied, $62.3 \%$ were women and $62.4 \%$ were over 40 years old. Most of the attendances (56.8\%) were held in the capital and in public services (70.3\%). The users were satisfied with the services, with a percentage that ranged from 79 to $90 \%$, according to the health regions. Men and people over 60 evaluated services more positively; the high complexity and the services of the capital had better results ( $p \leq 0.001$ ), as well as the private units. The importance of health evaluation and the relevance of analyzing, disseminating and giving continuity to the SUS Charter instrument is emphasized, with a view to subsidizing further studies and reflections on the quality of services.

KEYWORDS Health evaluation. Hospital care. Ambulatory care. Consumer behavior. 


\section{Introdução}

A partir da Constituição Federal de 1988, todos os brasileiros passaram a ter direito à saúde, com a criação do Sistema Único de Saúde (SUS), sendo dever do Estado provê-la, mediante políticas sociais e econômicas. Nesse contexto, a garantia da qualidade da assistência pública à saúde tornou-se um direito social do ser humano no País, cujo fortalecimento deu-se ao longo dos anos com o desenvolvimento de dispositivos de participação social, como as plenárias e os fóruns e resoluções de conselhos de saúde, as estratégias de educação permanente voltadas para o controle social, a Política Nacional de Gestão Estratégica e Participativa no SUS, entre outros ${ }^{1-3}$.

Nesse sentido, o conceito de saúde ganhou nova abrangência e passou a ser entendido como resultado das condições sociais e de vida. O direito à saúde e de acesso aos serviços passaram a ser reconhecidos como direitos de cidadania. Os princípios do SUS tornaram-se orientadores das práticas assistenciais, contemplando o acesso universal e igualitário, a regionalização, a hierarquização e a descentralização dos serviços de saúde, o atendimento na perspectiva da integralidade e da participação popular. Assim, o conhecimento do direito à saúde é um requisito fundamental para que $o$ usuário possa utilizar o serviço considerando os princípios que regem o SUS ${ }^{4,5}$.

Partindo-se da concepção do usuário como um membro corresponsável pela gestão do sistema, procura-se definir a institucionalização da avaliação dos serviços de saúde por meio do estímulo à disseminação de uma cultura avaliativa que torne essa prática incorporada às rotinas de gerenciamento no âmbito do sistema de saúde brasileiro. Logo, a prática avaliativa incorporada às rotinas de gerenciamento que envolva a perspectiva do usuário vem crescendo na última década, uma vez que a avaliação da satisfação do usuário constitui um dos mais importantes indicadores para mensurar a qualidade dos serviços de saúde, além de representar umas das razões principais para se trabalhar com a melhoria da qualidade ${ }^{3,6}$.

A criação do Departamento de Ouvidoria Geral do SUS (Doges), da Secretaria de Gestão Estratégica e Participativa (SGEP), foi um dos marcos importantes para viabilizar e coordenar a realização de estudos e pesquisas no campo da ouvidoria em saúde, com foco especial na participação do usuário. O Doges elabora, a partir de manifestações colhidas por meio de carta, internet, telefone e atendimento presencial, relatórios gerenciais ou temáticos e direciona-os para gestores e organismos de controle social?.

Uma das iniciativas resultantes da criação do Doges foi o instrumento Carta SUS, a qual constitui-se em um instrumento de transparência, avaliação e fiscalização com a principal finalidade de auxiliar o Ministério da Saúde (MS) na identificação de possíveis irregularidades na prestação de serviços. A partir de seu envio, em caráter de correspondência, os usuários do SUS podem conferir se os dados enviados pelos órgãos competentes correspondem aos serviços prestados, acompanhar os custos de sua internação e dos seus serviços, bem como possibilita a avaliação do atendimento ${ }^{8}$.

A Carta SUS está relacionada aos dados da Autorização de Internação Hospitalar (AIH) e da Autorização de Procedimento Ambulatorial de Alta Complexidade (Apac). Ambas contêm cartão-resposta anexado com perguntas sobre o atendimento recebido, a qualidade das equipes de saúde, o estado das instalações físicas e quanto à recomendação da unidade de saúde a um amigo ou familiar8 .

A taxa de alcance da estratégia no País (quadriênio 2012, 2013, 2014 e 2015) foi de, aproximadamente, $80 \%$, o que demonstra como essa sistemática pode contribuir para fortalecer a transparência dos atendimentos prestados pelos serviços públicos de saúde, além de incentivar a participação do cidadão na manifestação de possíveis irregularida$\mathrm{des}^{8}$. Nesse sentido, a Carta SUS foi escolhida em virtude de se configurar como uma das 
principais estratégias de obtenção da opinião dos usuários sobre os serviços do SUS e pelo fato de não se ter conhecimento de estudos analíticos consistentes baseados em sua utilização.

Ante o exposto e considerando a relevância de se trabalharem as informações advindas dos usuários dos serviços, objetivou-se analisar a satisfação dos usuários e a caracterização dos serviços de atendimento hospitalar e ambulatorial do SUS.

\section{Métodos}

Realizou-se um estudo transversal analítico por meio de dados de usuários que participaram do instrumento Carta SUS entre 2012 e 2013, no estado do Rio Grande do Norte (RN). Em 2010, o estado apresentou uma densidade demográfica de $59,99 \mathrm{hab} / \mathrm{km}^{2}$, com população composta de 3.168 .027 pessoas, distribuída em 167 municípios, de acordo com levantamento feito pelo Instituto Brasileiro de Geografia e Estatística (IBGE) ${ }^{9}$.

Entre 2012 e 2013, período disponibilizado pelo MS, foram enviadas aos usuários do Rio Grande do Norte 399.959 Cartas SUS. Considerando que 23,29\% foram devolvidas, estima-se que um total de 93.150 tenham sido respondidas. A partir da eliminação de usuários que responderam mais de uma vez à correspondência do MS, chegou-se a um número de 17.333 usuários, configurando-se como a amostra total do presente estudo.

Ressalta-se que as Cartas foram enviadas a todos os cidadãos que passaram por internação hospitalar ou procedimentos ambulatoriais de alta complexidade em hospitais/unidades de saúde públicas ou contratualizadas com o SUS. Em janeiro de 2013, meio do período em estudo, o RN contava com 3.584 estabelecimentos de saúde, estando assim distribuídos em suas regiões de saúde: 258 em São José de Mipibu (I), 416 em Mossoró(II), 255 em João Câmara (III), 426 em Caicó (IV), 169 em Santa Cruz (V), 347 em Pau dos Ferros (VI), 1.591 em Natal (VII) e 122 na região de saúde de Açu (VIII) ${ }^{\mathbf{1 0}}$.

$\mathrm{O}$ acesso aos dados foi obtido junto ao Departamento de Ouvidoria Geral do SUS/ Secretaria de Gestão Estratégica e Participativa (SGEP) do MS, considerando ser esse órgão o fiel depositário dessas informações. Prevendo a não utilização das informações confidenciais a que tiveram acesso para outro fim que não o proposto nesta pesquisa, os pesquisadores emitiram termo de confidencialidade e sigilo.

Para composição das variáveis do estudo, foram considerados todos os campos preenchidos pelos usuários na Carta SUS, disponibilizados pela SGEP. A distribuição das variáveis analisadas no presente estudo encontra-se apresentada no quadro 1.

Quadro 1. Tipo, natureza e conceito das variáveis pesquisadas no estudo, Carta SUS

\begin{tabular}{llll}
\hline Variável & Tipo & Natureza & Conceito \\
\hline Sexo & Independente & Categórica & Sexo do usuário: masculino ou feminino. \\
Idade & Independente & Categórica & Faixa etária do usuário. \\
Setor de gestão & Independente & Categórica & Estabelecimento público ou privado. \\
Local & Independente & Categórica & Localização do estabelecimento: capital ou interior. \\
Região de saúde & Independente & Categórica & Região de saúde na qual se localiza o estabelecimento. \\
Procedimento & Independente & Categórica & Procedimento realizado pelo usuário. \\
Complexidade & Independente & Categórica & Procedimento de alta ou média complexidade. \\
Autorização & Independente & Categórica & Indica se usuário foi submetido a uma AlH ou Apac. \\
\hline
\end{tabular}




\begin{tabular}{llll}
\hline Quadro 1. (cont.) & & & \\
\hline Estrutura física & Dependente & Categórica & $\begin{array}{l}\text { Avaliação do usuário com relação às instalações físicas } \\
\text { do estabelecimento. }\end{array}$ \\
Equipe de saúde & Dependente & Categórica & $\begin{array}{l}\text { Avaliação do usuário com relação ao atendimento } \\
\text { prestado pela equipe. }\end{array}$ \\
Tratamento & Dependente & Categórica & $\begin{array}{l}\text { Avaliação do usuário com relação à maneira como foi } \\
\text { tratado no hospital. }\end{array}$ \\
\hline
\end{tabular}

Fonte: Secretaria de Gestão Estratégica e Participativa (SGEP), Ministério da Saúde, 2014

O banco de dados foi construído preliminarmente no aplicativo de criação de planilhas eletrônicas Excel, o qual foi posteriormente exportado para o software SPSS (versão 17.0), no qual se realizou um processo de conferência e correção dos erros identificados por meio de dupla digitação. Uma vez consolidado o banco de dados, realizou-se análise estatística descritiva e inferencial buscando associação entre as variáveis independentes e as variáveis dependentes do estudo.

Os dados utilizados não identificam os usuários, atendendo às diretrizes éticas para a realização de pesquisas envolvendo seres humanos. Esta pesquisa foi apresentada ao Comitê de Ética em Pesquisa do Hospital Universitário Onofre Lopes (CEP-HUOL), sendo aprovada com parecer $n^{\circ} 834.621$, no dia 26 de setembro de 2014.

\section{Resultados}

Dos 17.333 usuários que responderam à Carta SUS no período de 2012 a 2013, no Rio Grande do Norte, $62,3 \%$ eram mulheres, $62,4 \%$ tinham acima de 40 anos, $56,8 \%$ dos atendimentos foram realizados na capital do estado, 70,3\% em serviços públicos, sendo a maior parte dos procedimentos $(68,7 \%)$ caracterizada como de média complexidade e realizada em âmbito hospitalar (73,3\%).

Com relação ao SUS, o estado encontra-se dividido em oito Regiões de Saúde (RS), em consonância com o Plano Diretor de Regionalização (PDR/RN). A RS VII (Metropolitana) é a que reúne o maior contingente populacional, concentrando $38,2 \%$ da população total do estado, enquanto a RS VIII (Vale do Açu), composta por 13 municípios, é a menos populosa, concentrando apenas $4,5 \%$ do total1 1 . 
Figura 1. Mapa com os percentuais de avaliações positivas dos usuários sobre os serviços de saúde de acordo com as regiões de saúde do estado, 2012-2013, RN-Brasil

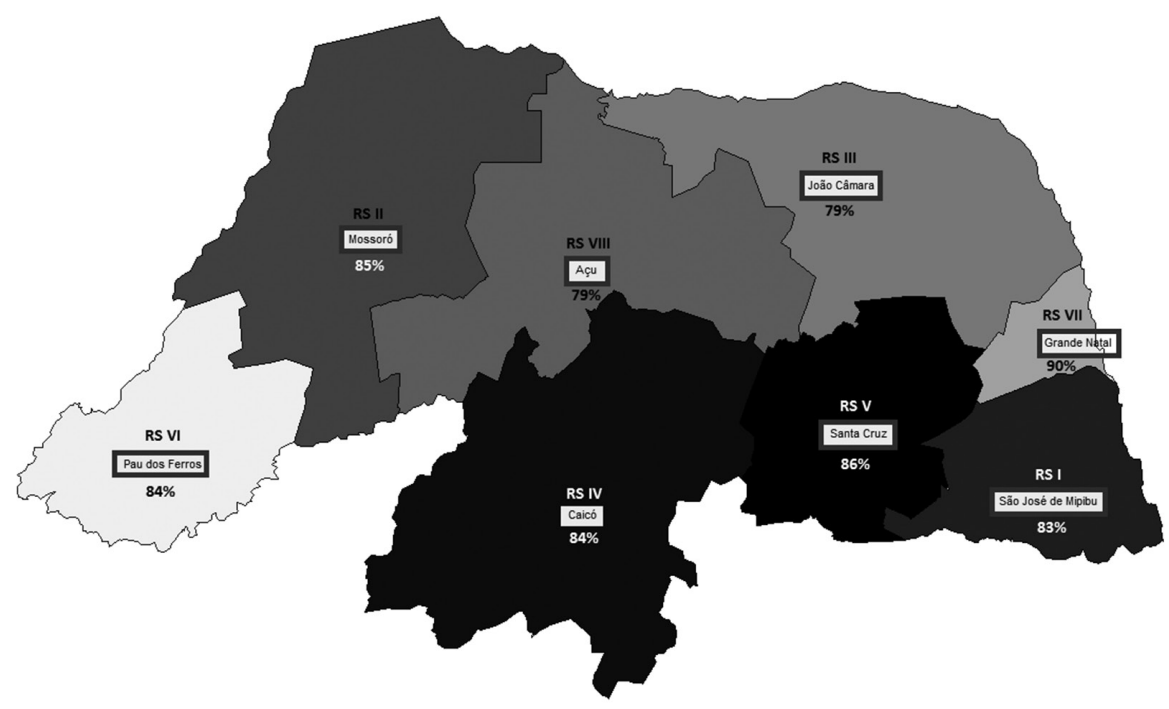

Fonte: Secretaria de Gestão Estratégica e Participativa (SGEP), Ministério da Saúde, 2014.

Conforme visto na figura 1, observaram-se patamares elevados de avaliação positiva, com maior destaque para as regiões V e VII, agreste e litoral oriental do estado, e menor desempenho das regiões III e VIII, com abrangência maior da região central ou do litoral norte. O nível de satisfação ficou acima de $80 \%$ em quase todas as regiões de saúde do estado.

De acordo com o evidenciado na tabela 1, os dados apresentaram relativo equilíbrio quanto à faixa etária, com menor número de usuários na faixa de 0 a 18 anos e maior quantidade de participantes na faixa etária $\geq 60$ anos.

Tabela 1. Informações sociodemográficas e características dos serviços de saúde, 2012-2013, RN, Brasil

\begin{tabular}{lrr}
\hline Variável & $\mathbf{n}$ & $\%$ \\
\hline Sexo $(\mathbf{n}=16.157)$ & & \\
\hline Feminino & 10.058 & 62,3 \\
Masculino & 6.099 & 37,7 \\
\hline Faixa etária (anos, $n=17.333)$ & 2.165 & 12,5 \\
\hline O a 18 & 4.352 & 25,1 \\
19 a 39 & 4.722 & 27,2 \\
40 a 59 & 6.094 & 35,2 \\
$\geq 60$ & & \\
\hline
\end{tabular}


Tabela 1. (cont.)

\begin{tabular}{lcr}
\hline Localização & & \\
\hline Capital & 9.842 & 56,8 \\
Interior & 7.491 & 43,2 \\
\hline Gestão administrativa & & \\
\hline Pública & 12.189 & 70,3 \\
Privada & 5.144 & 29,7 \\
\hline
\end{tabular}

Fonte: Secretaria de Gestão Estratégica e Participativa (SGEP), Ministério da Saúde, 2014.

Observou-se que os serviços estavam mais localizados na capital do estado, provavelmente pelo maior número de unidades nessa região, e a gestão administrativa concentrou a maior parte dos estabelecimentos na rede inteiramente pública. Por pertencer à área metropolitana do estado, a Região de Saúde VII compreendeu a maior quantidade de usuários que realizaram os procedimentos por meio da AIH e da Apac.

Por sua vez, a tabela 2 evidencia o nível de satisfação dos usuários no que diz respeito à qualidade da estrutura física, da equipe de saúde e do tratamento recebido nos serviços estudados. Para definição dos critérios, estabeleceu-se como adequada a junção das avaliações ‘bom' e ‘muito bom' e como inadequadas as avaliações 'regular', 'ruim' e 'muito ruim', sinalizadas na Carta SUS.

Do total de 17.333 usuários, 13.842 (81,4\%) consideraram a qualidade da estrutura física adequada, da mesma forma que 15.830 (91,9\%) sinalizaram, por meio da Carta SUS, que a qualidade das equipes de saúde dos serviços era adequada, ao mesmo tempo que a qualidade do tratamento recebido foi considerada adequada para $15.471(89,8 \%)$ usuários.

Entre outras evidências, a tabela 2 explicita que não houve diferença na avaliação dos serviços quando comparados homens e mulheres, com exceção do item estrutura física, no qual os homens avaliaram mais positivamente os serviços; as pessoas com 60 anos ou mais tendem a avaliar melhor as unidades de saúde, especialmente a equipe de saúde e a qualidade do tratamento; em todos os aspectos pesquisados, a alta complexidade e os serviços da capital tiveram melhores resultados ( $\mathrm{p} \leq 0,001)$, em detrimento da média complexidade e do interior, respectivamente; os usuários avaliaram mais positivamente o atendimento prestado pelas unidades de saúde conveniadas ao SUS do que os estabelecimentos públicos; por fim, os serviços vinculados à Apac obtiveram resultados mais satisfatórios quando comparados aos prestados pelas unidades relacionadas à AIH. 
Tabela 2. Associação entre os desfechos 'estrutura física', 'equipe de saúde' e 'tratamento' e as variáveis independentes utilizadas no estudo, 2012-2013, RN-Brasil

\begin{tabular}{|c|c|c|c|c|c|c|c|}
\hline \multicolumn{8}{|c|}{ Qualidade da estrutura física } \\
\hline \multirow[t]{2}{*}{ Variável } & \multicolumn{2}{|c|}{ Adequado } & \multicolumn{2}{|c|}{ Inadequado } & \multirow[t]{2}{*}{$\chi^{2^{\star}}$} & \multirow[t]{2}{*}{$\mathbf{p}^{\star \star}$} & \multirow[t]{2}{*}{$O R^{\star \star \star}$} \\
\hline & $\mathrm{n}$ & $\%$ & $n$ & $\%$ & & & \\
\hline \multicolumn{8}{|l|}{ Sexo } \\
\hline Masculino & 5029 & 82,5 & 1070 & 17,5 & 28,48 & $\leq 0,001$ & $1,24(1,15-1,35)$ \\
\hline Feminino & 7947 & 79,0 & 2111 & 21,0 & & & \\
\hline \multicolumn{8}{|l|}{ Idade } \\
\hline$\geq 60$ anos & 5076 & 83,3 & 1018 & 16,7 & 68,97 & $\leq 0,001$ & $1,40(1,29-1,52)$ \\
\hline$<60$ anos & 8766 & 78,0 & 2473 & 22,0 & & & \\
\hline \multicolumn{8}{|c|}{ Estabelecimento } \\
\hline Outro tipo & 3172 & 80,8 & 2487 & 18,9 & 2,76 & $<0,097$ & $1,07(0,98-1,18)$ \\
\hline Hospital & 10670 & 79,6 & 2737 & 20,4 & & & \\
\hline \multicolumn{8}{|c|}{ Local de atendimento } \\
\hline Capital & 8261 & 83,9 & 1581 & 16,1 & 235,3 & $\leq 0,001$ & $1,78(1,65-1,92)$ \\
\hline Interior & 5581 & 74,5 & 1910 & 25,5 & & & \\
\hline \multicolumn{8}{|c|}{ Complexidade } \\
\hline Alta & 3879 & 90,6 & 404 & 9,4 & 448,1 & $\leq 0,001$ & $3,15(2,82-3,51)$ \\
\hline Média & 8960 & 75,3 & 2940 & 24,7 & & & \\
\hline \multicolumn{8}{|l|}{ Gestão } \\
\hline Privada & 4456 & 86,6 & 688 & 13,4 & 208,1 & $\leq 0,001$ & $1,93(1,76-2,11)$ \\
\hline Pública & 9386 & 77,0 & 2803 & 23,0 & & & \\
\hline \multicolumn{8}{|c|}{ Autorização } \\
\hline APAC & 4033 & 87,2 & 594 & 12,8 & 209,3 & $\leq 0,001$ & $2,00(1,82-2,20)$ \\
\hline $\mathrm{AlH}$ & 9809 & 77,2 & 2897 & 22,8 & & & \\
\hline \multicolumn{8}{|c|}{ Qualidade da equipe de saúde } \\
\hline \multirow[t]{2}{*}{ Variável } & \multicolumn{2}{|c|}{ Adequado } & \multicolumn{2}{|c|}{ Inadequado } & $\chi^{2^{\star}}$ & $p^{\star \star}$ & $O R^{\star \star \star}$ \\
\hline & $\mathrm{n}$ & $\%$ & $n$ & $\%$ & & & \\
\hline \multicolumn{8}{|l|}{ Sexo } \\
\hline Masculino & 5590 & 91,7 & 509 & 8,3 & 0,480 & $<0,489$ & $1,04(0,92-1,16)$ \\
\hline Feminino & 9187 & 91,3 & 871 & 8,7 & & & \\
\hline \multicolumn{8}{|l|}{ Idade } \\
\hline$\geq 60$ anos & 5607 & 92,0 & 487 & 8,0 & 5,485 & $<0,019$ & $1,14(1,02-1,28)$ \\
\hline$<60$ anos & 10223 & 91,0 & 1016 & 9,0 & & & \\
\hline \multicolumn{8}{|c|}{ Estabelecimento } \\
\hline Outro tipo & 3539 & 90,1 & 387 & 9,9 & 9,01 & $<0,003$ & $0,83(0,73-0,93)$ \\
\hline Hospital & 12291 & 91,7 & 1116 & 8,3 & & & \\
\hline \multicolumn{8}{|c|}{ Local de atendimento } \\
\hline Capital & 9213 & 93,6 & 629 & 6,4 & 149,5 & $\leq 0,001$ & $1,93(1,73-2,15)$ \\
\hline Interior & 6617 & 88,3 & 874 & 11,7 & & & \\
\hline
\end{tabular}


Tabela 2. (cont.)

\begin{tabular}{|c|c|c|c|c|c|c|c|}
\hline \multicolumn{8}{|c|}{ Complexidade } \\
\hline Alta & 4092 & 95,5 & 191 & 4,5 & 146,7 & $\leq 0,001$ & $2,55(2,18-2,98)$ \\
\hline Média & 10634 & 89,4 & 1266 & 10,6 & & & \\
\hline \multicolumn{8}{|l|}{ Gestão } \\
\hline Privada & 4751 & 92,4 & 393 & 7,6 & 9,82 & $<0,002$ & $1,21(1,07-1,36)$ \\
\hline Pública & 11079 & 90,9 & 1110 & 9,1 & & & \\
\hline \multicolumn{8}{|c|}{ Autorização } \\
\hline APAC & 4301 & 93,0 & 326 & 7,0 & 21,06 & $\leq 0,001$ & $1,34(1,18-1,53)$ \\
\hline $\mathrm{AlH}$ & 11529 & 90,7 & 1177 & 9,3 & & & \\
\hline \multicolumn{8}{|c|}{ Qualidade do tratamento } \\
\hline \multirow[t]{2}{*}{ Variável } & \multicolumn{2}{|c|}{ Adequado } & \multicolumn{2}{|c|}{ Inadequado } & \multirow[t]{2}{*}{$\chi^{2^{\star}}$} & \multirow[t]{2}{*}{$\mathbf{p}^{\star \star}$} & \multirow[t]{2}{*}{$O R^{\star \star \star}$} \\
\hline & n & $\%$ & $\mathrm{n}$ & $\%$ & & & \\
\hline \multicolumn{8}{|l|}{ Sexo } \\
\hline Masculino & 5501 & 90,2 & 598 & 9,8 & 2,04 & $<0,152$ & $1,08(0,97-1,20)$ \\
\hline Feminino & 9001 & 89,5 & 1057 & 10,5 & & & \\
\hline \multicolumn{8}{|l|}{ Idade } \\
\hline$\geq 60$ anos & 5541 & 90,9 & 553 & 9,1 & 21,4 & $\leq 0,001$ & $1,28(1,15-1,42)$ \\
\hline$<60$ anos & 9965 & 88,7 & 1274 & 11,3 & & & \\
\hline \multicolumn{8}{|c|}{ Estabelecimento } \\
\hline Outro tipo & 3513 & 89,5 & 413 & 10,5 & 0,002 & $<0,961$ & $1,00(0,89-1,12)$ \\
\hline Hospital & 11993 & 89,5 & 1414 & 10,5 & & & \\
\hline \multicolumn{8}{|c|}{ Local de atendimento } \\
\hline Capital & 9024 & 91,7 & 818 & 8,3 & 120,0 & $\leq 0,001$ & $1,71(1,55-1,89)$ \\
\hline Interior & 6482 & 86,5 & 1009 & 13,5 & & & \\
\hline \multicolumn{8}{|c|}{ Complexidade } \\
\hline Alta & 4040 & 94,3 & 243 & 5,7 & 160,7 & $\leq 0,001$ & $2,41(2,10-2,78)$ \\
\hline Média & 10389 & 87,3 & 1511 & 12,7 & & & \\
\hline \multicolumn{8}{|l|}{ Gestão } \\
\hline Privada & 4677 & 90,9 & 467 & 9,1 & 16,58 & $\leq 0,001$ & $1,25(1,12-1,40)$ \\
\hline Pública & 10829 & 88,8 & 1360 & 11,2 & & & \\
\hline \multicolumn{8}{|c|}{ Autorização } \\
\hline APAC & 4246 & 91,8 & 381 & 8,2 & 35,60 & $\leq 0,001$ & $1,43(1,27-1,61)$ \\
\hline $\mathrm{AlH}$ & 11260 & 88,6 & 1446 & 11,4 & & & \\
\hline
\end{tabular}

Fonte: Secretaria de Gestão Estratégica e Participativa (SGEP), Ministério da Saúde, 2014

${ }^{*}$ Qui-quadrado, ${ }^{* *}$ Valor de $\mathrm{p},{ }^{* * *}$ Odds Ratio. 
De modo geral, os dados estudados revelaram que há mais serviços de alta complexidade na capital do que no interior. Os hospitais encontraram-se mais na capital, bem como há mais serviços de alta complexidade públicos na capital do que no interior do estado, levando-se em consideração os serviços pesquisados. Ainda, os dados mostraram que a maior parte dos serviços foi autorizada pela AIH na média complexidade e no interior, e pela Apac na alta complexidade e entre os serviços localizados na capital.

\section{Discussão}

Ao se avaliar a qualidade dos serviços de saúde, tanto os da esfera pública como os da esfera privada, existem controvérsias relacionadas às formas adequadas de se mensurar os níveis de satisfação dos usuários, seja na sua percepção referente aos serviços recebidos ou no impacto dos atributos geradores dessa satisfação. Todavia, há consenso que a satisfação do usuário ocorre quando suas necessidades e expectativas são atendidas, o que leva a resultados positivos acerca dos serviços de saúde analisados. Desse modo, a necessidade de avaliação em saúde que incorpore o olhar dos usuários torna-se imperativa na área da saúde ${ }^{12-15}$.

Sob essa ótica, torna-se necessária a análise da satisfação dos usuários, com o objetivo principal de conhecer as percepções deles no tocante aos processos envolvidos no atendimento em saúde. Assim sendo, para que ocorra maior precisão, é imprescindível o monitoramento de parâmetros de qualidade relacionados à infraestrutura, aos processos de trabalho e aos resultados vinculados aos cuidados de saúde prestados, a fim de atingir a maior satisfação do usuário ${ }^{\mathbf{1 2}, 16}$. Portanto, esses parâmetros levam em conta as premissas da teoria de Donabedian, o qual, em 1978, já preconizava que a avaliação dos serviços de saúde deveria considerar estes três aspectos: estrutura, processo e resultado, e que em todos a satisfação do usuário deveria estar em foco ${ }^{17}$.
Este estudo constatou que houve diferenças importantes entre os graus de satisfação dos usuários quando comparadas as regiões de saúde do estado. Um estudo sobre a avaliação da cobertura do desempenho dos programas de controle de tuberculose em Santa Catarina também identificou diferenças entre as regiões de saúde do estado. Nessa perspectiva, quando as regiões de saúde foram analisadas isoladamente e comparadas umas às outras, percebeu-se uma grande variedade de nichos de gravidade distribuídos entre diversas delas, assim como distintos empenhos da gestão administrativa para seu enfrentamento ${ }^{18}$.

Ainda por esse prisma, um estudo que comparou os achados das regiões de saúde do estado do Rio Grande do Norte no que se refere à avaliação da mortalidade por câncer do colo do útero, no período de 1996 a 2010, observou que a Região Metropolitana, a que possui melhor condição socioeconômica, apresentou tendência decrescente nas taxas de mortalidade por essa patologia específica, ao contrário da ascendência dos números das interioranas ${ }^{19}$.

Outro achado deste estudo mostrou que as mulheres estiveram mais presentes do que os homens nos serviços. Estudos corroboram o índice de maior participação feminina e exclusão dos homens pelos serviços de saúde. Estes se apresentam em um contexto de atendimento inadequado a esse público, com pouco interesse em propiciar espaços que o homem possa adentrar, o que pode aumentar as suas dificuldades na procura por assistência e colabora para um menor empenho desses sujeitos em manter hábitos saudáveis e adesão aos tratamentos nas situações de risco ${ }^{20-23}$.

Em outra perspectiva, percebeu-se, pelos achados deste estudo, que os usuários com 60 anos ou mais tendem a avaliar melhor as unidades de saúde, especialmente as equipes de saúde e a qualidade do tratamento. Isso pode ser um reflexo dos interesses desse público, pois a forma de ver o mundo e o lugar social em que se encontram pode influenciar diretamente a avaliação dos serviços de saúde. Se no decorrer da vida esses idosos não tiveram um 
acesso adequado às unidades de saúde, o que está sendo oferecido na atualidade pode estar em padrões satisfatórios para eles, mas não necessariamente representa uma comprovação da efetividade das políticas públicas de saúde ${ }^{15}$.

Nesse enquadramento, embora a assistência integral seja princípio basilar do SUS, um estudo realizado no interior de São Paulo, em 2014, verificou que alguns idosos consideraram o atendimento oferecido mais próximo à dádiva e não ao direito à saúde ${ }^{24}$. Desse modo, a facilidade do acesso aos serviços nos tempos atuais, tendo em vista que antigamente só tinha direito quem pagava previdência, contribui para o vínculo do idoso com a rede de serviços e a consequente avaliação positiva; além disso, a ocorrência da aposentadoria conduz ao tédio e fortalece a interação dos idosos nos grupos de convivência dos serviços de saúde 24-26.

Sob outro ângulo, esta pesquisa revelou que a qualidade da estrutura física teve menos avaliações positivas dos usuários do que os itens equipe de saúde e tratamento recebido. Tal resultado é corroborado por outros estudos, os quais demonstram que a qualidade da estrutura física é um dos aspectos mais mencionados na avaliação dos serviços de saúde, principalmente na ótica dos usuários. As instalações físicas de um serviço podem afetar drasticamente a qualidade do cuidado, uma vez que interferem na assistência prestada pela equipe ao desmotivar profissionais e levar desconforto e situações antiéticas aos usuários 27-29.

Ressalta-se que os serviços da esfera privada, em geral, possuem melhores condições de infraestrutura, o que pode influenciar a avaliação dos usuários. Ademais, cabe destacar que há uma ideologia arraigada acerca da qualidade do serviço público, visto, muitas vezes, como de menor qualidade em comparação com a iniciativa privada. Essas questões são importantes e devem ser levadas em consideração quando se avalia a satisfação dos usuários ${ }^{30}$.

Os usuários desta pesquisa avaliaram mais positivamente o atendimento prestado pelas unidades de saúde privadas conveniadas ao SUS do que pelas públicas. Tal achado é consoante com outros estudos os quais identificam divergências na relação entre público e privado $^{31}$. Uma pesquisa realizada no estado do Mato Grosso constatou que a relação público-privada no âmbito da atenção ambulatorial é complexa e nebulosa. Nesse cenário, apesar de a legislação do SUS definir elementos essenciais para o alcance de seus princípios, a prática evidencia permissão de 'espaços' para que o setor privado se insira e seja beneficiado pelo Estado, somando-se a isso os conflitos de poder político e econômico entre os grupos de interesse ${ }^{32}$.

Ainda, autores do estudo feito no Mato Grosso, supracitado, afirmam que as entidades de representação privada queixam-se dos baixos valores de reembolso predefinidos na tabela SUS, mas sobrevivem economicamente do aporte financeiro de recursos públicos, tendo em vista que aproximadamente dois terços de sua clientela são de usuários do SUS. Tais autores acrescentam que a maior concentração de leitos para internação esteja nas regiões mais ricas, onde também se encontra a maior parte dos beneficiários de planos de saúde. Certamente, a desigualdade de distribuição dos serviços e a não complementariedade dos segmentos público e privado afetam a oferta, que, por sua vez, é refletida no acesso aos serviços de saúde, ou seja, não parece haver coordenação entre os segmentos público e privado ${ }^{32}$.

Os dados desta pesquisa apontaram, ainda, para a avaliação positiva da alta complexidade e dos serviços da capital em detrimento da média complexidade e dos estabelecimentos do interior. Tais achados estão de acordo com vários estudos, os quais observam a concentração de serviços de alta complexidade em grandes centros urbanos e a alta satisfação dos usuários com os serviços de saúde privados e de maior complexidade ${ }^{33-35}$.

Reconhece-se que, apesar da evolução das políticas de saúde e dos avanços na terapêutica em todo o País, o manejo da assistência e na prevenção das doenças é passível de fragilidades regionais de tecnologia, recursos e estruturas relacionadas à coordenação das ações e dos serviços de saúde. Nesse sentido, concebe-se atribuir as taxas elevadas de satisfação nas 
regiões metropolitanas ao maior nível de densidade tecnológica e ao maior número de profissionais com conhecimentos especializados nessas regiões, as quais se constituem como polos historicamente mais desenvolvidos em termos tecnológicos, de tratamento e na realização de pesquisas científicas em saúde ${ }^{33-35}$.

Desse modo, depreende-se que persistem realidades nas quais há desigualdades de oferta de serviços, tanto em questões de acesso como de qualidade e efetividade, especialmente quando se comparam capitais com municípios interioranos. Assim, muitos usuários são prejudicados ao terem que se deslocar do interior à capital em busca de suprirem suas necessidades em saúde. Tais iniquidades representam fatores estruturais associados ao isolamento geográfico e político, bem como à baixa capacidade de gestão e arrecadação dos municípios menores, o que impede o avanço tecnológico nos seus serviços e colabora para a perpetuação da baixa resolutividade e de níveis de saúde aquém dos desejados ${ }^{36}$.

Por outro lado, autores defendem que o consumo elevado de tecnologia dura, em regra, pouco ou nada acrescenta ao processo de produção da saúde da população. Para eles, os profissionais médicos tendem a subestimar o contato e o diálogo com o usuário, solicitam uma bateria de exames laboratoriais e/ou complementares, bem como a realização de consultas especializadas, atividades que poderiam ser resolvidas na própria atenção primária. Por sua vez, de acordo com esses autores, o usuário tende a valorizar a qualidade do seu atendimento pelos procedimentos complexos utilizados em seu tratamento; ou seja, a alta complexidade tende a ser mais bem avaliada quando comparada aos demais níveis. Nesse contexto, algumas hipóteses são levantadas: a importância atribuída pelos usuários aos serviços especializados mais complexos e o fato de os usuários ficarem satisfeitos simplesmente por conseguirem o acesso a esse tipo de serviço, independentemente de fatores como o tempo de espera ${ }^{33,34}$.

Além das discussões supracitadas, destaca-se a questão da resolutividade, a qual pode ser uma forte influência na percepção positiva dos usuários sobre os serviços referentes à alta complexidade. Estudos constataram que o aumento do nível de complexidade geralmente interfere na procura dos usuários pelos serviços, uma vez que esses ainda sejam fortemente orientados pelo modelo de atenção de queixa-conduta dos casos agudos, mesmo tais casos sendo passíveis de resolução em níveis de complexidade menores ${ }^{31,37-39}$.

Outros autores vão além ao afirmarem que a demanda dos problemas apresentados pela população pede uma gestão de cuidados com maior organização das ações. Torna-se necessário buscar mais recursos físicos, tecnológicos e humanos que possam contemplar as questões de vulnerabilidade e complexidade dos processos de adoecimento. Nesse sentido, o caminho para compor um sistema de saúde resolutivo e acolhedor não descarta a necessidade de consultas com médicos especialistas, de atendimentos com outros profissionais, de procedimentos diagnósticos e terapêuticos e de internações hospitalares, mas a construção e a valorização das relações interpessoais e interdisciplinares e da comunicação entre os envolvidos precisam ser otimizadas no SUS ${ }^{38-}$ 40. Nessa perspectiva, a plena organização da Rede de Atenção à Saúde (RAS) é fundamental tanto para o desenvolvimento das ações coletivas e preventivas como para a garantia da complementariedade das atividades que exigem maior grau de complexidade tecnológica dos serviços ${ }^{\mathbf{4 1}}$.

Por fim, ressalta-se que o elevado grau de satisfação dos usuários com os serviços, evidenciado por esta pesquisa, é corroborado por outros estudos, embora se considere o nível baixo de expectativas com relação aos direitos sociais e ao padrão de qualidade por parte dos usuários. Apesar dos aspectos deficitários enfrentados pelo serviço público de saúde, como dificuldade de acesso, baixa resolutividade, entre outros, os usuários entendem que o sistema público consegue cumprir sua função de oferecer serviços de saúde. Para os autores desses estudos, as avaliações tidas 
como negativas do ponto de vista dos usuários devem ser consideradas com foco na melhoria dos serviços, enquanto as positivas representam estímulos à atuação dos profissionais e gestores, bem como à defesa do serviço público ${ }^{\mathbf{4 2 , 4 3}}$.

Entre as limitações do estudo, ressaltam-se: a pouca devolutiva dos usuários referente à Carta SUS ao se considerar o número total de correspondências enviadas; o fato da amostra de respondentes desse instrumento possivelmente não abranger indivíduos que não tenham alfabetização ou não consigam, por quaisquer motivos, retorná-la; um possível viés de colinearidade de variáveis dependentes, uma vez que na capital se concentram os serviços especializados que foram os mais bem avaliados; a não explicitação do percentual de cartas respondidas por região, tendo em vista que isso pode influenciar a composição do universo do estudo.

\section{Considerações finais}

A importância da avaliação dos serviços de saúde é inequívoca. Além disso, a utilização das informações provenientes de tais avaliações é indispensável para o aperfeiçoamento e a melhoria da qualidade do atendimento prestado. Este estudo mostrou, principalmente, altos índices de avaliação positiva dos serviços de saúde públicos ou conveniados ao SUS por parte dos usuários, com abrangência de questões sobre estrutura física, equipe de saúde e o tratamento prestado pelos estabelecimentos. Todavia, a estrutura física, a média complexidade, os serviços públicos e do interior receberam as avaliações mais negativas por parte dos usuários.

A ferramenta Carta SUS mostrou-se capaz de subsidiar estudos e reflexões necessárias sobre a qualidade de serviços hospitalares e ambulatoriais pertencentes ou conveniados à rede pública. Todavia, os autores deste estudo não obtiveram informações sobre a continuidade dessa estratégia no País, com ausência de informações nas comunicações oficiais do Governo. Entende-se que a continuidade dessa estratégia tornar-se-ia relevante no âmbito da avaliação do serviço público de saúde, haja vista a possibilidade, por exemplo, de produção de séries históricas que oportunizariam análises de tendências de satisfações dos usuários.

Nesse contexto, a falta de informação por parte dos órgãos públicos competentes a respeito da continuidade dessa estratégia reforça a ideia de uma avaliação em saúde meramente burocrática, com base em processos incipientes e sem aplicação prática. Ou seja, as ferramentas para avaliação são criadas, mas não são incorporadas, de fato, às rotinas permanentes de gestores, gerentes e todos os atores envolvidos no setor.

\section{Colaboradores}

Silva Junior DNS, Braga BSF, Santos CDP, Borges REA e Noro LRA contribuíram para a concepção e planejamento, análise e interpretação dos dados, participação na revisão crítica do conteúdo e participação da aprovação da versão final do manuscrito. 


\section{Referências}

1. Macêdo HJR, Macêdo AFF, Sousa MNA. Conhecimentos de alunos da educação de jovens e adultos sobre seus direitos enquanto usuários do SUS. C\&D Rev. Eletrônica da Fainor. 2014; 7(1):199-210.

2. Seleghim MR, Teixeira JÁ, Matsuda LMIK. Avaliação de usuários sobre a qualidade dos serviços de um pronto-socorro. Rev. Rene Fortaleza. 2010; 11(3):12231.

3. Guizardi FL. A autocrítica necessária: notas sobre os desafios do controle social na saúde. Rev. Eletrônica Comum. Informação Inovação em Saúde [internet]. 2007 [acesso em 2018 nov 12]; 9(3):1-7. Disponível em: https://www.reciis.icict.fiocruz.br/index. php/reciis/article/view/1028/pdf1028.

4. Fertonani HP, Pires DEP, Biff D, et al. Modelo assistencial em saúde: conceitos e desafios para a atenção básica brasileira. Ciênc. Saúde Colet. [internet]. 2015 [acesso em 2018 nov 12]; 20(6):1869-1878. Disponível em: http://www.scielo.br/pdf/csc/v20n6/1413-8123csc-20-06-1869.pdf.

5. Brasil. Carta dos direitos dos usuários da saúde. Ministério da Saúde. 4. ed. 2013.

6. Paiva MBP, Mendes W, Brandão AL, et al. Uma contribuição para a avaliação da Atenção Primária à Saúde pela perspectiva do usuário. Physis Rev. Saúde Coletiva [internet]. 2015 [acesso em 2018 nov 12]; 25(3):925950. Disponível em: http://www.scielo.br/pdf/physis/v25n3/0103-7331-physis-25-03-00925.pdf.

7. Brasil. Ministério da Saúde. Participação e Controle Social [internet]. 2018 [acesso em 2018 nov 12]. Disponível em: http://portalms.saude.gov.br/participacao-e-controle-social/ouvidoria-do-sus

8. Brasil. Carta SUS. Ministério da Saúde: 2018.

9. Instituto Brasileiro de Geografia e Estatística. Panorama Rio Grande do Norte. IBGE [internet]. 2010 [acesso em 2018 nov 13]. Disponível em: https://ci- dades.ibge.gov.br/brasil/rn/panorama.

10. Brasil. Ministério da Saúde - Cadastro Nacional dos Estabelecimentos de Saúde do Brasil CNES [internet]. 2013 [acesso em 2018 nov 13]. Disponível em: http:// tabnet.datasus.gov.br/cgi/tabcgi.exe?cnes/cnv/estabrn.def.

11. Brasil. Secretaria de Estado da Saúde Pública do Rio Grande do Norte. Plano Estadual de Saúde: PES 20162019 [internet]. 2016 [acesso em 2018 nov 13]. Disponível em: http://adcon.rn.gov.br/ACERVO/sesap/ DOC/DOC000000000142450.PDF.

12. Castillo L, Dougnac A, Vicente I, et al. Los predictores de satisfacción de pacientes en un centro hospitalario universitario. Rev. Med. Chil. [internet]. 2007 [acesso em 2018 nov 14]; 135(6):696-701. Disponível em: https://scielo.conicyt.cl/pdf/rmc/v135n6/art02. pdf.

13. Cruz WBS, Melleiro MM. Análise da satisfação dos usuários de um hospital privado. Rev. Esc. Enferm. USP [internet]. 2010 [acesso em 2018 nov 14]; 44(1):147-53. Disponível em: http:// www.scielo.br/scielo.php?script=sci_arttext\&pid =S0080-62342010000100021.

14. Silva ER, Hecksher SD. Qualidade do atendimento em serviços públicos de saúde. Rev. Eletrônica Gestão Saúde. 2016; 7(1):980-92.

15. Akerman M, Nadanovsky P. Evaluation of Health Services - What to Evaluate? Cad Saúde Pública. 1992; 8(4):361-365.

16. Alghamdi FS. The impact of service quality perception on patient satisfaction in government hospitals in southern Saudi Arabia. Saudi Med J. 2014 out; 35(10):1271-1273.

17. Reis EJFB, Santos FP, Campos FE et al. Avaliação da qualidade dos serviços de saúde: notas bibliográficas. Cad. Saúde Pública [internet]. 1990 [acesso em 
2018 nov 13]; 6(1):50-61. Disponível em: http://www. scielo.br/pdf/csp/v6nl/v6nla06.pdf.

18. Franco SC, Mendonça SA. Avaliação do risco epidemiológico e do desempenho dos programas de controle de tuberculose nas Regiões de Saúde do estado de Santa Catarina, 2003 a 2010. Epidemiol. Serv. Saúde. 2015; 24(1):59-70.

19. Sousa AMV, Souza DLB. Mortalidade por câncer do colo do útero no estado do Rio Grande do Norte, no período de 1996 a 2010: tendência temporal e projeções até 2030. Epidemiol. Serv. Saúde [internet]. 2016 [acesso em 2018 nov 13]; 25. Disponível em: https:// www.scielosp.org/pdf/ress/2016.v25n2/311-322/pt.

20. Levorato CD, Mello LM, Silva AS, et al. Fatores associados à procura por serviços de saúde numa perspectiva relacional de gênero. Ciênc. Saúde Colet. [internet]. 2014 [acesso em 2018 nov 13]; 19(4):12631274. Disponível em: http://www.scielo.br/pdf/csc/ v19n4/1413-8123-csc-19-04-01263.pdf.

21. Silva SPC, Menandro MCS. As representações sociais da saúde e de seus cuidados para homens e mulheres idosos. Saúde e Soc. [internet]. 2014 [acesso em 2018 nov 13]; 23(2):626-40. Disponível em: http:// www.scielo.br/pdf/sausoc/v23n2/0104-1290-sausoc-23-2-0626.pdf.

22. Cortez MB, Machado NM, Trindade ZA, et al. Profissionais de saúde e o (não)atendimento ao homem-pai: Análise em representações sociais. Psicol. em Estud. 2016; 21(1):53-63.

23. Barreto MS, Arruda GOMS. Como os homens adultos utilizam e avaliam os serviços de saúde. Rev. Eletr. Enf. [internet]. 2015[acesso em 2018 nov 13]; 17(3). Disponível em: https://www.revistas.ufg.br/fen/article/view/29622/20772.

24. Vello LS, Popim RC, Carazzai EM, et al. Saúde do Idoso: percepções relacionadas ao atendimento. Esc. Anna Nery [internet]. 2014 [acesso em 2018 nov 13]; 330-335. Disponível em: http://www.scielo.br/pdf/ ean/v18n2/1414-8145-ean-18-02-0330.pdf.
25. Melo LP, Campos EA. "O grupo facilita tudo": significados atribuídos por pacientes portadores de diabetes mellitus tipo 2 a grupos de educação em saúde. Rev. Latino-Am. Enferm. [internet]. 2014 [acesso em 2018 nov 13]; 22(6). Disponível em: http://www.scielo. br/pdf/rlae/v22n6/pt_0104-1169-rlae-22-06-00980. pdf.

26. Wichmann FMA, Couto AN, Areosa SVC, et al. Grupos de convivência como suporte ao idoso na melhoria da saúde. Rev. Bras. Geriatr. e Gerontol. [internet]. 2013 [acesso em 2018 nov 13]; 16(4):821-32. Disponível em: http://www.scielo.br/pdf/rbgg/vl6n4/18099823-rbgg-16-04-00821.pdf.

27. Souza LS, Rocha JKS, Martins KAF et al. A qualidade do atendimento prestado pelos pronto-socorros de hospitais públicos do Brasil. Rev. Adm. Hosp. e Inovação em Saúde [internet]. 2013 [acesso em 2018 nov 13]. Disponível em: http://revistas.face.ufmg.br/ index.php/rahis/article/view/2129/1367.

28. Leite SNMF, Álvares J, Guerra Junior AAC, et al. Infraestrutura das farmácias da atenção básica no Sistema Único de Saúde: Análise dos dados da PNAUM-Serviços. Rev. Saúde Pública [internet]. 2017 [acesso em 2018 nov 13]; 51. Disponível em: http://www.rsp.fsp. usp.br/wp-content/uploads/articles_xml/0034-8910rsp-S1518-51-s2-87872017051007120/0034-8910-rspS1518-51-s2-87872017051007120-pt.x83902.pdf.

29. Silva ALA, Mendes ACG, Miranda GMD, et al. A qualidade do atendimento ao parto na rede pública hospitalar em uma capital brasileira: a satisfação das gestantes. Cad. Saúde Pública [internet]. 2017 [acesso em 2018 nov 13]; 33(12). Disponível em: http:// www.scielo.br/pdf/csp/v33n12/1678-4464-csp-3312-e00175116.pdf.

30. Arruda CAM, Bosi MLM. Satisfação de usuários da atenção primária à saúde: um estudo qualitativo no Nordeste do Brasil. Interface - comum. Saúde, Educ. [internet]. 2017; 21(61):321-32. Disponível em: http:// www.scielo.br/pdf/csp/v33n12/1678-4464-csp-3312-e00175116.pdf.

31. Tabassum T, Ashraf M, Thaver I. Hospitalized Pa- 
tients' Awareness of Their Rights-A Cross Sectional Survey in A Public And Private Tertiary Care Hospitals of Punjab, Pakistan. J Ayub Med Coll Abbottabad. 2016; 28(3):582-586.

32. Romano CMC, Scatena JHG, Kehrig RT. Articulação público-privada na atenção ambulatorial de média e alta complexidade do SUS: Atuação da Secretaria de Estado de Saúde de Mato Grosso. Physis. 2015; 25(4):1095-1115.

33. Starfield B. Atenção Primária - Equilíbrio entre necessidades de saúde, serviços e tecnologia. Brasília, DF: UNESCO; Ministério da Saúde. 2002.

34. Coelho MO, Jorge MSB. Tecnologia das relações como dispositivo do atendimento humanizado na atenção básica à saúde na perspectiva do acesso, do acolhimento e do vínculo. Ciênc. Saúde Colet. [internet]. 2009 [acesso em 2018 nov 13]; 14:1523-1531. Disponível em: http://www.scielo.br/scielo.php?script=sci_ arttext\&pid=S1413-81232009000800026\&lng=pt\&n $\mathrm{rm}=\mathrm{iso} \& \mathrm{tlng}=\mathrm{pt}$.

35. Schramm JMDA, Oliveira AF, Leite IDC, et al. Transição epidemiológica e o estudo de carga de doença no Brasil. Ciênc. Saúde Colet. 2004; 9(4):897-908.

36. Garnelo L, Sousa ABL, Silva CO. Regionalização em Saúde no Amazonas: avanços e desafios. Ciênc. Saúde Colet. 2017; 22(4):1225-1234.

37. Machado JP, Cristina A, Martins M. Avaliação da qualidade do cuidado hospitalar no Brasil: uma revisão sistemática. Cad. Saúde Pública. 2013; 29(6):10631082.

38. Kneipp MM, Cardoso CM, Beatriz S, et al. Serviços de urgência e emergência: quais os motivos que levam o usuário aos pronto-atendimentos? Saúde (Santa Maria) [internet]. 2015 [acesso em 2018 nov 13]; 41(1):195-
200. Disponível em: https://periodicos.ufsm.br/index.php/revistasaude/article/viewFile/15061/pdf.

39. Nobre CLC, Oliveira A, Joia JH, et al. O trabalho em rede nos casos de alta complexidade e de alta vulnerabilidade: a experiência de uma UBS. Distúrb. comum. [internet]. 2017 [acesso 2018 nov 13]; 29(2):377384. Disponível em: https://revistas.pucsp.br/index. php/dic/article/view/30703/23009.

40. Pessôa LR, Santos IS, Machado JP, et al. Realocar a oferta do SUS para atender problemas do futuro: o caso do trauma no Brasil. Saúde debate [internet]. 2016 [acesso em 2018 nov 14]; 40(110):9-19. Disponível em: http://www.scielo.br/pdf/sdeb/v40n110/01031104-sdeb-40-110-0009.pdf.

41. Brasil. Ministério da Saúde. Portaria n ${ }^{\circ} 4.279$, de 30 de dezembro de 2010. Estabelece diretrizes para a organização da Rede de Atenção à Saúde no âmbito do Sistema Único de Saúde (SUS). Diário Oficial da União. 31 Dez 2010. 88-93.

42. Castro HCO, Machado LZ, Walter MIMT, et al. A Satisfação dos Usuários com o Sistema Único de Saúde (SUS). Sociedade em Debate [internet], 2008 [acesso em 2018 nov 13]; 14(2):113-34. Disponível em: http://www.rle.ucpel.tche.br/index.php/rsd/article/ view/375.

43. Ricci NA, Wanderley FS, Oliveira MS, et al. O hospital-escola de São Carlos: análise do funcionamento por meio da satisfação dos usuários. Ciênc. Saúde Colet. [internet]. 2011 [acesso em 2018 nov 13]; 16(1):1125-1134. Disponível em: http:// www.scielo.br/scielo.php?script=sci_arttext\&pid $=$ S1413-81232011000700044.

Recebido em 30/05/2018 Aprovado em 23/09/2018

Conflito de interesses: inexistente

Suporte Financeiro: não houve 